\title{
ALEJANDRO TOMASINI, LECTOR DE WITTGENSTEIN EN MÉXICO
}

\author{
Carolina Rodríguez Rodríguez \\ Universidad Santo Tomás
}

\section{Resumen}

Este texto constituye un informe parcial de la investigación en torno a la Recepción de la filosofía analítica en América Latina, y se dedica a explorar los aportes particulares que ha realizado Alejandro Tomasini Bassols en la comprensión y difusión de la obra de Ludwig Wittgenstein en los países de habla hispana, especialmente en México.

Teniendo como marco metodológico la perspectiva de la historia de las ideas, el artículo llega a establecer que las contribuciones de Tomasini a la interpretación de la obra de Wittgenstein son 1. La redefinición disciplinar de la filosofía, 2. El esclarecimiento de la naturaleza del método analítico, 3. La aplicación del método analítico al análisis de problemas filosóficos y culturales particulares, 4. El desarrollo de una filosofía analítica de la religión, y 5. El esbozo preliminar de las condiciones para una filosofía analítica latinoamericana. Como es evidente, estos resultados van más allá de la exégesis de Wittgenstein y se proyectan hacia una aplicación de la reflexión analítica a temas de interés latinoamericano.

\section{Palabras clave}

Wittgenstein, filosofía analítica, método analítico, lenguaje.

\begin{abstract}
The text includes a partial inform on the investigation regarding the Reception of the analytical philosophy in Latin America, and is dedicated to explore the particular contributions that have been made by Alejandro Tomasini Bassols in the understanding and spreading of Ludwig Wittgenstein's work in Spanish speaking countries, specially in Mexico.
\end{abstract}


Taking as methodological frame the perspective of the history of ideas, the article comes to establish Tomasini's contributions to the interpretation of Wittgenstein's work. These contributions are the following: 1 . The disciplinary redefinition of the philosophy, 2 . The clearing up of the nature of the analytical method, 3. The application of the analytical method to the analysis of particular cultural and philosophical problems, 4. The development of an analytical philosophy of the religion, 5. The preliminary rough draft of the conditions for an analytical Latin America philosophy. As it is evident, these results go beyond Wittgenstein's exegesis and are projected towards and application of the analytical reflection to topics that call Latin America interest.

\section{Index terms}

Wittgenstein, analytical philosophy, analytical method, language.

\section{Presentación del proyecto}

Este proyecto pertenece al grupo de investigación Fray Bartolomé de las Casas y se inscribe en la Línea de recepción de corrientes. Pretende realizar un balance de la recepción de la filosofía analítica en México y, en particular, del pensamiento de Wittgenstein. Dada su extensión, este es un proyecto a largo plazo que, en una primera fase, se dedica a la reconstrucción del pensamiento de uno de los principales exponentes del pensamiento analítico en la actualidad: Alejandro Tomasini Bassols.

Como enfoque metodológico se asume la historia de las ideas, porque permite ir más allá del problema de la originalidad del pensamiento latinoamericano, para poner de manifiesto las condiciones a partir de las cuáles se ha hecho posible la recepción de los diferentes autores y tendencias europeas, analizando cuál fue el contexto propicio para este transplante, sus efectos, la posibilidad de nuevos desarrollos, etc.

\section{Antecedentes}

El país de América Latina en el que la filosofía analítica ha tenido una mayor expansión es México, pionero en la recepción desde la década del 40 hasta hoy. Testimonio de esto es la cooperación permanente entre la Sociedad Argentina de Análisis Filosófico (SADAF) y la UNAM, la traducción de textos analíticos clásicos y la publicación de los resultados de investigaciones en dos revistas analíticas especializadas, Dianoia y Crítica, la realización de congresos y eventos internacionales, etc.

El principal antecedente del trabajo aquí propuesto lo constituye la antología publicada por Jorge Gracia bajo el título El análisis filosófico en América Latina ${ }^{1}$. Esta obra, editada en 1985, realizó una selección por países de los textos analíticos más importantes en nuestro continente hasta 1985, y además introdujo una presentación del desarrollo de la historia de las ideas analíticas en cada país, a cargo de un pensador representativo de esta

1 GRACIA, Jorge. El análisis filosófico en América Latina. México: F.C.E., 1985. 
corriente. En el caso mexicano, fue Enrique Villanueva el encargado de esta tarea, quien en su momento destacó la labor desarrollada por figuras como José Gaos, Eduardo García Maynéz, Luis Villoro, Fernando Salmerón y Alejandro Rossi, entre otros. Frente a la reconstrucción del caso mexicano, el trabajo de Villanueva es el único que se conoce en su género.

Sin embargo, y aunque se trata de un importante antecedente, el texto de Villanueva publicado hace 19 años no da cuenta de los desarrollos actuales de la analítica en este país, hecho que justifica y legitima la necesidad de realizar un trabajo de balance que desde la historia de las ideas realice una reconstrucción del pensamiento analítico durante todo el proceso de recepción. Por otra parte, es necesario mencionar como antecedente la recopilación que sobre lógica se hizo en la base de datos del Instituto de Investigaciones Filosófica de la $\mathrm{UNAM}^{2}$, que constituye un trabajo bibliográfico valioso, pero que no avanza a una reconstrucción panorámica desde la historia de las ideas.

Por otra parte, Gabriel Vargas Lozano escribe una panorámica de la filosofía mexicana en el siglo XX, en donde la filosofía del lenguaje apenas sí aparece mencionada como una de las tendencias actuales que ha dejado sentir su influencia. No obstante, el autor señala la importancia de "distinguir entre lo que implica mera recepción de las teorías y aportación creativa”3.

Otro precedente lo constituye el texto de $L a$ filosofía analítica en Colombia ${ }^{4}$, en el cual se realiza un balance del desarrollo de esta tendencia de pensamiento en el periodo comprendido entre 1960 y 2000. El objetivo fue presentar una visión de conjunto, teniendo en cuenta los principales autores, categorías, textos y problemas analíticos trabajados. Finalmente, el último antecedente lo representa el artículo de Felix Valdés García "Panorama de la filosofía analítica latinoamericana” ${ }^{5}$, dedicado a realizar una reconstrucción panorámica de la recepción del análisis filosófico en América Latina, siguiendo las líneas canónicas propuestas por Jorge Gracia.

\section{Aportes de Tomasini al análisis, a partir de su lectura de Wittgenstein}

Alejandro Tomasini Bassols es doctor en
humanidades por la Universidad de Varso-
via, con la tesis El atomismo lógico de
Bertrand Russell (1982), y master en filoso-
fía de la Universidad de Oxford con el traba-
jo Estudio comparativo entre los atomismos

2 En 1970, el Instituto de Investigaciones Filosóficas de la UNAM empezó a publicar la Bibliografía filosófica mexicana, con el propósito de reunir los trabajos que se publican en México sobre filosofía. Después de nueve volúmenes, en 1979, se suspendió su publicación. Años más tarde en, 1989, al empezar una segunda etapa se optó por procesar sus datos y crear con ellos una base denominada FILOS, tomada como fuente de la Bibliografía filosófica mexicana. De esta segunda etapa se han publicado nueve volúmenes correspondientes a los años 1986-1994. La base de datos FILOS registra los datos bibliográficos de los documentos sobre filosofía publicados en México a partir de 1986. En el primer número de Modus ponens se usó a FILOS para proporcionar un panorama de la producción en lógica en México. Más de cien registros selectos demostraron que hay intensa actividad en este campo.

3 VARGAS LOZANO, Gabriel. “Esbozo histórico de la filosofía mexicana del siglo XX”. En: Filosofía de la cultura en México. Coord., Mario Teodoro Ramírez. México: Universidad Michoacana de San Nicolás de Hidalgo / Editorial Plaza y Valdés, 1997.

4 RODRÍGUEZ, Carolina. La filosofía analítica en Colombia. Bogotá: El Búho, 2002.

5 VALDÉS GARCÍA, Félix. "Panorama de la filosofía analítica latinoamericana”. En: Filosofía en América Latina. La Habana: Editorial Félix Varela, 1998, pp. 339-369. 
lógicos de Wittgenstein y Russell (1984). Además, es investigador titular de tiempo completo y definitivo del Instituto de Investigaciones Filosóficas de la UNAM, miembro del Sistema Nacional de Investigadores y miembro de la Asociación Filosófica de México. De su trabajo como traductor se destacan las versiones españolas de textos fundamentales para la literatura analítica como Investigaciones filosóficas y observaciones sobre los colores, de Wittgenstein.

El interés de Tomasini gira en torno al pensamiento de Bertrand Russell y Ludwig Wittgenstein. Avanza de un rusellianismo radical, en donde trabajó principalmente el atomismo, hacia la filosofía del segundo Wittgenstein. Sin duda alguna, uno de sus objetivos filosóficos consiste realizar en español análisis gramaticales (cuyos paradigmas están desde luego en las obras del segundo Wittgenstein) al modo en que Norman Malcolm y Morris Lazerowitz han mostrado que se pueden realizar. Su filosofía de la religión es un claro ejemplo de ello.

El aporte de Tomasini al análisis filosófico es muy amplio. Para efectos de esta exposición me centraré en temas como las nuevas perspectivas en la comprensión de la obra de Wittgenstein, la revisión de la tarea del filosofar, el esclarecimiento conceptual en torno al método analítico, los desarrollos de una filosofía analítica de la religión y el proyecto de una filosofía analítica latinoamericana.

\subsection{Nuevas perspectivas en la comprensión de la obra de Wittgenstein}

Me parece muy importante dejar claro en este punto que en sus primeras obras Tomasini desarrolló un trabajo divulgativo e incluso exegético frente al análisis, en particular de las filosofías de Russell y Wittgenstein. No obstante, el autor ha logrado separarse de visiones canónicas o tradicionalistas para promover lecturas heréticas, indicando nuevas posibilidades de comprensión y aplicación del pensamiento de Wittgenstein, así:

1. En la discusión en torno a Wittgenstein se identifican dos periodos diferenciados en su pensamiento, de modo que el debate tiende a establecer si hay solución de continuidad o una ruptura total entre ellos. La propuesta de Tomasini es que el pensamiento del autor se desarrolla a través de tres fases cuyas coyunturas están representadas por 1. el Tractatus logico philosophicus, 2. los Cuadernos azul y marrón y 3. las Investigaciones filosóficas. Entre estas fases existe coherencia y continuidad, de manera que es posible rastrear un planteamiento y desarrollo común de los problemas filosóficos.

2. Interpreta la noción de objeto en el Tractatus de una manera no fisicalista al resaltar sus elementos apriorísticos y trascendentales ${ }^{6}$.

3. Plantea y desarrolla el debate entre Wittgenstein y Chomsky a propósito de la relación lenguaje-pensamiento, defendiendo, como resulta lógico, una interpretación pragmática de esta relación.

4. Relaciona el Tractatus de Wittgenstein con el pensamiento de Heráclito.

5. Subraya la diferencia entre los atomismos de Russell y Wittgenstein.

6. Como un hecho derivado de lo anterior, propone el carácter no formalista del pri-

6 Cf. TOMASINI, Alejandro. "Dos nociones de objeto en el Tractatus”. En: Analogía (México), jul.-dic., 1993. 
mer Wittgenstein. Para Tomasini, formalistas son Frege y Russell, incluso Husserl, pero nunca Wittgenstein.

7. Sugiere una interpretación conductista del modo operativo en el que se dan los sistemas de aprendizaje y entrenamiento al interior de los juegos del lenguaje. Para esto tiene en cuenta la relación entre signo, sensación y reacción.

\subsection{Tarea de la filosofía}

Tomasini explica la evolución de la historia de la filosofía a partir de la perspectiva del lenguaje y la construcción de conceptos. Sin embargo, aunque la tarea fundamental del filósofo ha sido la construcción de edificios conceptuales, generalmente no lo ha hecho en condiciones de rigor, claridad y precisión, tanto desde el aspecto lógico como desde el gramatical. Esto ha vulnerado la imagen profesional del filósofo, ya que se le cuestiona, ridiculiza y descalifica por el carácter ilusorio de los problemas que plantea y por el tipo de respuestas estériles que emprende.

A partir de lo anterior, la filosofía analítica está llamada, desde la perspectiva de Tomasini, a convertirse en una instancia crítica con respecto al pensamiento tradicional, poniendo de manifiesto los errores en el uso del lenguaje de una manera metafilosófica. Esto implica abandonar las tentativas de generalización y explicación, porque la filosofía no enseña nada, simplemente describe, ejemplificando las dificultades filosóficas a partir de situaciones específicas:

Las disquisiciones metafilosóficas pueden ser sumamente ilustrativas pues, en- tre otras cosas, exigen que los diversos puntos que se vayan desarrollando queden siempre debidamente ejemplificados, esto es, que se vayan extrayendo y examinando los casos particulares y los ejemplos concretos de todas las ramas de la filosofía tradicional ${ }^{7}$.

La tarea del filósofo es la descripción del lenguaje natural a partir de un conjunto de casos y ejemplos que puedan poner en claro la forma en la que actúan las reglas y el uso de las expresiones en sus contextos respectivos. Hay que disolver la apariencia de profundidad, descartar el ansia de generalidad, para que el filósofo pueda desarrollar una tarea verdaderamente útil.

El papel del nuevo tipo de filósofo no es ya el de inventar nuevas teorías, doctrinas, etc., sino, más bien, el de convertirse en una especie de policía intelectual: cada vez que alguien dice algo filosófico -que es algo por lo que tenemos una fuerte inclinación-, hay que hacerle ver que está, por así decirlo, cometiendo una infracción, que está descuidando o que no se ha fijado en algún mecanismo o aspecto de esa inmensa maquinaria que es el lenguaje natural ${ }^{8}$.

La función de la filosofía es elucidatoria, actuando como un disolvente de cuestionamientos. Ahora bien, esto exige luchar con problemas filosóficos concretos y luego sí hacer metafilosofía; no es posible descartar la filosofía de un plumazo. Es necesario tomarse el trabajo de reflexionar sobre un conjunto delimitado de problemas para no caer en el facilismo y la charlatanería. Me parece claro que Tomasini no nos

7 Cf. TOMASINI, Alejandro. “¿Qué fue de la filosofía analítica?”. En: Analogía (México), jul.- dic., 1999, p. 35.

8 TOMASINI, Alejandro. “Reflexiones wittgenstenianas sobre el lenguaje”. En: Anuario de letras (México), Tomo XXV, 1987, p. 144. 
invita a abandonar la filosofía sino a entender el peso que han tenido sus debates y problemas, sus respuestas fallidas, para luego sí disolverlas mediante el análisis. Esta es una actitud muy responsable, porque un paso previo a la disolución está en la meditación, y eso hace de la filosofía una disciplina ex-postfacto.

\subsection{Aplicación del método analítico}

Richard Rorty ${ }^{9}$ argumenta, no sin razón, que la filosofía analítica presume de claridad y, sin embargo, ha incurrido en la paradoja de carecer de precisión a la hora de definir sus conceptos fundamentales, entre ellos el de método analítico. Sin recurrir a una simplificación que aspire a disolver la diversidad, considero que Tomasini se ha hecho cargo de esta objeción formulada por Rorty al presentar una caracterización evolutiva del método analítico a lo largo de los autores más representativos; reconoce por lo menos cuatro versiones de este método ${ }^{10}$ :

a. Modelo del análisis lógico: realizado a partir de la teoría de las descripciones de Russell.

b. Modelo del análisis conceptual: llevado a cabo por Moore, desde la perspectiva del sentido común.

c. Modelo del análisis filosófico: desarrollado por Carnap al emplear la paráfrasis como forma de disolver un problema filosófico.

d. Modelo de las definiciones recursivas: este método, empleado por Tarski, busca construir una teoría semántica de la verdad. e. Modelo del análisis gramatical: método empleado por Wittgenstein para esclarecer el modo en que un conjunto de reglas gobiernan el uso de una expresión determinada.

A Tomasini no le basta con arrojar luz frente a una perplejidad habitual dentro de los círculos analíticos al no tener una claridad sobre las implicaciones del método y sus posibles aplicaciones. Toma partido y decide que el método de análisis gramatical es el más adecuado para asumir la labor de esclarecimiento del lenguaje filosófico. Lo interesante es que no se limita a reconstruir el método, a describirlo o ilustrar operatividad a partir de los ejemplos wittgenstenianos clásicos. El mismo Tomasini aplica el método a problemas filosóficos tradicionales al desarrollar la actividad metafilosófica:

La investigación gramatical tiene como objetivo sacar a la luz reglas que de hecho rigen el uso significativo de las expresiones que, por otra parte, los filósofos, y en general, los hablantes en sus momentos filosóficos, presuponen aunque mal emplean ${ }^{11}$.

En este contexto, el autor ofrece ilustración de cómo aplicar el método analítico en la revisión de problemas clásicos, analizando la estructura misma de las preguntas y la viabilidad de las respuestas, que generalmente no eliminan el interrogante sino que lo complican. Un interesante caso de aplicación del método está dado en la evaluación que hizo Tomasini del debate desarrollado entre Copleston y Russell a propósito de la posibilidad de probar la existencia de Dios desde una argumentación lógica ${ }^{12}$. Para ello tuvo

9 Cf. RORTY, Richard. La filosofía y el espejo de la naturaleza. Madrid: Tecnos, 1988.

10 TOMASINI, Alejandro. ¿Qué fue de la filosofía analítica? En: Analogía (México), julio-diciembre, 1999, p. 12.

11 TOMASINI, Alejandro. Filosofía: la naturaleza de los conceptos. México: UNAM, 1998.

12 Cf. TOMASINI, Alejandro. “Copleston, Russell y la existencia de Dios”. En: Analogía (México), juliodiciembre, 1987. 
en cuenta las falencias y debilidades de los interlocutores al argumentar a partir de conceptos como el de razón suficiente, causa, necesidad y existencia.

Otro ejemplo importante lo constituye el examen sistemático que realiza de un problema muy viejo y trajinado en el pensamiento occidental: el de la existencia del mundo externo. Muestra el inconveniente mismo de formular la relación entre el mundo externo y los sense-data, rebate las diferentes soluciones dadas al respecto y, a partir de la imposibilidad de un lenguaje privado, disuelve la verdadera relevancia de la cuestión, echando así por tierra las diferentes aproximaciones que racionalistas, empiristas, fenomenistas y filósofos de la mente han desarrollado desde una perspectiva gnoseológica $^{13}$.

\subsection{Filosofía analítica de la religión}

A partir de los últimos desarrollos de la escuela de Oxford, la filosofía de la religión se ha convertido en un asunto fundamental para la reflexión analítica. Posturas clásicas como el agnosticismo de Russell o el neopositivismo de Carnap y Ayer fueron hasta hace poco tiempo canónicas, y despachaban la cuestión de la religión como si de un pseudoproblema se tratase. No obstante, las investigaciones de Ian Crombie incitan a:

...defender que la existencia de rasgos paradójicos permiten la elusividad en el lenguaje religioso. Ésta se manifiesta a través de usos y reglas particulares por parte de los creyentes, idea que le lleva a aproximar la elusividad lingüís- tica con la existencia de juegos de lenguaje de carácter religioso (...). Los cambios epistemológicos al interior del movimiento analítico han tenido una repercusión importante en el tipo de posiciones que se asumen ante la validez de los enunciados religiosos. Cuando la tendencia analítica mostró una impronta marcadamente cientificista, la discusión gravitó en torno a los criterios de verificación y falsación. Sin embargo, la revolución analítica generada por el segundo Wittgenstein y los filósofos de Oxford produjo un verdadero giro pragmático en el tratamiento de la religión y de su lenguaje ${ }^{14}$.

Ian Crombie y John Hick son los principales exponentes de la escuela de Oxford que influyen en el modo en el que Tomasini trabaja el tema de la religión. El objetivo consiste en desplazar las posturas atomistas y fisicalistas en aras del predominio de una concepción pragmática del lenguaje, que permita entender la religión con un juego de lenguaje válido, sometido a reglas particulares y que construye significados desde su interior.

Por esta razón, más que probar o negar la existencia de Dios desde una perspectiva analítica, el trabajo que se debe emprender es el de establecer qué se quiere decir cuando se habla de Dios y qué acciones se desarrollan públicamente en concordancia con esta creencia. Desde una perspectiva wittgensteniana, Tomasini deja de considerar a Dios como un concepto ostensivo, porque no se trata de un objeto empírico que

13 Cf. TOMASINI, Alejandro. "Reflexiones wittgenstenianas sobre el lenguaje”. En: Anuario de letras (México), Tomo XXV, 1987.

14 RODRÍGUEZ, Carolina. "La epistemología y el lenguaje como expresiones de ateísmo y agnosticismo en el pensamiento filosófico contemporáneo”. En: IX Congreso Internacional de Filosofía Latinoamericana. Bogotá: Biblioteca Colombiana de Filosofía, 2003, p. 241. 
pueda hallarse en un estado de cosas. En virtud de esto, Tomasini concluye que apelar a un concepto único de referencia es erróneo, de manera que es ilícito seguir aplicando las normas y criterios del juego de lenguaje científico al religioso; asumir la experiencia religiosa desde un enfoque formalista constituye un esfuerzo inútil, porque de un modo similar al de Ladriére ${ }^{15}$, el autor defiende la independencia en el nivel comprensivo del ámbito formal y el experiencial.

Deseo resaltar que Alejandro Tomasini es el único autor latinoamericano que se ha dedicado al estudio analítico de la religión. No se limita a ser un continuador de la labor emprendida por sus maestros de Oxford, dado que aplica la concepción wittgensteniana de juego de lenguaje a la comprensión de las formas de vida religiosa particulares y situadas, lo cual constituye un novedoso aporte no sólo para la tradición analítica, dedicada hasta ahora a teorizar en abstracto, sino también a la reconstrucción de la idiosincrasia y de la identidad cultural del pueblo mexicano. El aporte se ha orientado, sobre todo, a describir rituales, significados y prácticas religiosas asociadas con la Virgen de Guadalupe.

\subsection{Proyecto de una filosofía analítica latinoamericana}

En diferentes textos Tomasini ha asumido la reflexión en torno a las razones del colapso de la filosofía analítica. Dentro de la literatura especializada este tópico es frecuente y se relaciona con la crisis del positivismo lógico, la falta de claridad frente al método analítico, la pérdida de los límites disci- plinares ante saberes como la lógica y la lingüística, la falta de respuestas frente a problemas filosóficos convencionales, etc. Por ejemplo, autores como Richard Rorty o Juan José Acero argumentan que la filosofía analítica clásica constituye un campo de conocimiento sin nuevas posibilidades de exploración y avance. Quienes objetan el análisis filosófico consideran que se trata de un campo de trabajo finiquitado y caduco, enfrascado en problemas tan insustanciales y estériles como los que critica. Resultaría ser un modo de escolástica tardía y decadente que, dado su colapso, seguramente no reviste ningún interés para su recepción en nuestro contexto.

No obstante ello, cuando Tomasini se refiere a la crisis de la filosofía analítica no alude a las debilidades internas de esta tendencia, sino que más bien desde la óptica de la historia de las ideas describe cómo hechos externos - por ejemplo, la Segunda Guerra Mundial_-, generaron una diáspora en los centro de investigación analítica (Viena, Polonia, Berlín), de manera que las figuras más representativas de estos círculos se exiliaron en los Estados Unidos e Inglaterra, lugares donde desarrollaron una actividad filosófica por fuera de la ortodoxia analítica original.

Los que hoy se autodefinen como filósofos analíticos no lo son tales y sus productos no tienen nada que ver con el programa inicial. Así, mientras Tomasini identifica la filosofía analítica genuina con el pensamiento europeo, a la vez relaciona la versión espúrea con desarrollos asociados a tendencias típicamente norteamericanas que, como explica Borradori ${ }^{16}$, serían básicamente el

15 LADRIÈRE, Jean. La articulación del sentido. Salamanca: Sígueme, 2001. Ver el capítulo 2, "El lenguaje de la fe", y el 3, "Filosofía, ciencia y fe".

16 Cf. BORRADORI, Giovanna. Conversaciones filosóficas: El nuevo pensamiento norteamericano. Colección Vitral. Bogotá: Editorial Norma, 1989. 
pragmatismo de Dewey y el conductismo skineriano. Ahora bien, mientras que Borradori relaciona este sustrato primigenio como la condición de posibilidad para salir de la clausura analítica, Tomasini interpreta que el contacto con escuelas extrañas fue lo que generó un viraje hacia los problemas continentales.

Si ya no queda nada de la analítica inicial y el pensamiento norteamericano ha avanzado hacia la producción de propuestas que pueden calificarse como espúreas, pero al mismo tiempo como creadoras, en este esfuerzo Tomasini encuentra un camino a seguir para poder consolidar una filosofía analítica latinoamericana; al respecto, el autor declara que:

...la filosofía analítica puede todavía ser el Ave Fénix capaz de hacer florecer la investigación filosófica en los países de habla española y llevarla a las cumbres por las que hasta ahora muy pocos viajeros hispanohablantes se han aventurado ${ }^{17}$.

Retomando preocupaciones manifestadas por autores como Gaos y Zea, considero que Tomasini cree en la posibilidad de llegar a desarrollar un pensamiento original y propio que vaya más allá de la simple repetición y difusión de doctrinas extrañas. Si de lo que se trata es de dar lugar a un pensamiento propositivo, "la condición para el florecimiento de una escuela analítica latinoamericana es que la filosofía se practique directamente en español" ${ }^{18}$. Al hablar simultáneamente de pensamiento analítico y latinoamericano, Tomasini no nos está invitando a realizar una fusión ecléctica entre dos proyectos aparentemente fallidos que generan sospecha en varios círculos intelectua- les; más bien, de lo que se trata es de articular dos posibilidades que pueden ser de una fecundidad intelectual insospechada.

En un tono similar al de la generación técni$c a$, Tomasini le apuesta a la posibilidad de un pensamiento propio, teniendo como condiciones antecedentes las exigencias de rigor y claridad que, por supuesto, puede aportar la analítica. De esta manera, la perspectiva analítica nos provee como latinoamericanos de interesantes puntos de vista a desarrollar desde la metafilosofía, y sobre todo, de un método de trabajo preciso y riguroso, que le dé altura académica a nuestros debates. Tomasini señala que la riqueza del enfoque analítico frente al pensamiento filosófico latinoamericano permite:

...incorporar sus métodos de trabajo, aprender a aplicar técnicas de tratamiento de problemas, interiorizar su natural enfoque de las dificultades, educarnos en cierto espíritu de discusión libre y desprejuiciada y luego, lo más difícil, discutir en nuestro propio idioma sobre nuestros propios problemas ${ }^{19}$.

Además de lo anterior, Tomasini señala una segunda cualidad del enfoque analítico que como latinoamericanos podemos aprovechar. Si la filosofía es ante todo un ejercicio de construcción y análisis de los conceptos, es necesario que avancemos hacia la construcción de un corpus categorial propio. Esta necesidad, aunque sin adscribirla a una orientación analítica, ya había sido planteada por Dussel.

Así, al hablar del proyecto de una filosofía analítica latinoamericana, Tomasini enfatiza en su función metodológica para el plantea-

17 Cf. TOMASINI, Alejandro. “¿Qué fue de la filosofía analítica?”. En: Analogía (México), jul.- dic., 1999, p. 37.

$18 \quad$ Ibíd., p. 57.

19 Ibíd., p. 57. 
miento y esclarecimiento de problemas viables y significativos, en sus aportes técnicos, instrumentales y heurísticos, y en la posibilidad de un pensamiento propio articulado a partir de conceptos nuevos. Puede existir una filosofía analítica en nuestro contexto tomando el español como lengua que nos provea de casos y ejemplos cercanos; también puede darse si se enfoca la discusión de temas específicos desde el análisis.

Aunque Tomasini formula el proyecto de una analítica latinoamericana de una manera apenas preliminar, él mismo empieza a desarrollarlo. Apropiándose de las líneas generales del método de análisis gramatical propuesto por Wittgenstein, nos provee de nuevos ejemplos que van más allá de la ortodoxia tradicional, ejemplos que en mucho pueden igualar e incluso superar a los suministrados por los textos exegéticos clásicos. Los ejemplos de Tomasini suelen referirse a situaciones cotidianas, a casos de la filosofía de la aritmética y a eventos relacionados con el aprendizaje infantil. Por otra parte, el autor logra enfocar problemas analíticos convencionales desde perspectivas novedosas, como por ejemplo el de la existencia de Dios.

\section{Conclusiones}

Sin lugar a dudas, el principal aspecto que llama la atención en la obra de Alejandro Tomasini está dado en su intención de creatividad. Si bien de este autor no puede decirse que sea el creador de una filosofía original, tampoco puede asumirse que se limite a ser un repetidor. Aunque no ha llegado al nivel de originalidad de autores latinoamericanos de filiación analítica como Héctor Neri Castañeda o Tomás Moro Simpson, cuyas obras representan ya un aporte nuevo frente al debate filosófico europeo, cabe esperar que pueda llegar a emular estos avances.
Más aún, es preciso señalar que el esfuerzo desarrollado por Tomasini va más allá de la divulgación didáctica o de la reconstrucción exegética de problemas y categorías en algún autor específico. Nuestro autor conoce profundamente la tradición analítica y se atreve a realizar aproximaciones a ella desde la perspectiva de la historia de las ideas; ha realizado su propia versión acerca de su origen, desarrollo y decadencia, indicando elementos sugerentes para el desarrollo de un pensamiento analítico original en América Latina, y nos ha aportado un valioso modelo para la aplicación del método analítico a una amplia gama de problemas.

\section{ANEXO: Publicaciones de Alejandro Tomasini en filosofía analítica}

\section{Libros}

1) Los atomismos lógicos de Russell y Wittgenstein (México: Instituto de Investigaciones Filosóficas, UNAM, 1986).

2) El pensamiento del último Wittgenstein (México: Trillas, 1988).

3) Filosofía de la religión. Análisis y discusiones (México: Colofón, 1992). 2 ª edición (corregida) México: Grupo Editorial Interlínea, 1995.

4) Una introducción al pensamiento de Bertrand Russell (Zacatecas: Universidad Autónoma de Zacatecas, 1992).

5) Lenguaje y anti-metafísica. cavilaciones wittgensteinianas (México: Grupo Editorial Interlínea/INBA, 1994).

6) Ensayos de filosofía de la psicología (Guadalajara: Universidad de Gua-dalajara, 1995).

7) Enigmas filosóficos y filosofía wittgensteiniana (México: Grupo Editorial In- 
terlínea, 1996). $2^{\text {a }}$ edición (México: Édere, 2002).

8) Significado y denotación. la polémica Russell-Frege. Prólogo, selección, traducción y notas de ATB (México: Grupo Editorial Interlínea, 1996).

9) Editor de Cuentos religiosos de León Tolstoy (México: Grupo Editorial Interlínea, 1997).

10) Nuevos ensayos de filosofía de la religión (México: JGH Editores, 1999).

11) Teoría del conocimiento clásica y epistemología wittgensteiniana (México: Plaza y Valdés, 2001).

12) Estudios sobre las filosofías de Wittgenstein (México: Plaza y Valdéz, 2003).

\section{Artículos}

1) “Russell y el infinito”. En: Casa Abierta al Tiempo (UAM). Vol. II, No 114 (1981), México.

2) “'Tiene sentido la vida?”. En: La Palabra y el Hombre № 142 (1982), México.

3) "Wittgenstein y los argumentos trascendentales”. En: Revista de Filosofía № 155 (1986), México.

4) "Formas de vida y formación de conceptos”. En: Ciencia y Desarrollo No 168 (1986), México.

5) "La comprensión de las prácticas: el caso de la magia”. En: Investigación Humanística (UAM). № 12 (1986), México.

6) “La doctrina russelliana del pensamiento". En: Dianoia (1986), México.

7) “Aspectos de la filosofía de la psicología de Wittgenstein”. En: Revista Mexicana de Análisis de la Conducta № 13 (1986), México.

8) "Reflexiones wittgensteinianas sobre el lenguaje”. En: Anuario de Letras, Tomo XXV (1987), UNAM, México.
9) "La experiencia visual y la noción de ver como”. En: Revista Latinoamericana de Filosofía Vol. XIII, № 13 (1987), Argentina.

10) “Marx y Wittgenstein: filosofía y sociedad”. En: Revista de Filosofía № 15859 (1987), México (Hay traducción al polaco. Studia Filozoficzne, № 112, (1987), Polonia).

11) "Copleston, Russell y la existencia de Dios”. En: Analogía Año I, No II (1987), México.

12) "El Lenguaje de los sueños y la naturaleza de lo onírico”. En: Revista Mexicana de Análisis de la Conducta. № 112 (1987), México.

13) “Presentación de la traducción de las Philosophische Untersuchungen de Ludwig Wittgenstein”. En: Diánoia (1988), México.

14) “Historia de la filosofía: ¿Para qué?”. En: Diánoia (1988), México.

15) “En torno a la Ley del Tercero Excluido". En: Analogía, Año III, No II (1989), México.

16) "La filosofía mística de Russell y lo indecible en el Tractatus”. En: Diánoia (1990), México.

17) "Conceptos religiosos y vida religiosa". En: Dianoia. Número Especial dedicado a Ludwig Wittgenstein (1989), México.

18) "El antropocentrismo lingüístico de Ludwig Wittgenstein”. En: Sociológica (UAM), № 114 (1990), México.

19) “Aporías, antinomias y el infinito; la crítica de Russell a Zenón y Kant”. En: Mathesis Vol. VI, № 13 (1990), México.

20) “Contrafácticos”. En: Analogía. Año IV N 12 (1990), México.

21) “Lenguaje y pensamiento: enfoques wittgensteinianos”. En: Cuaderno de Filosofía No 115. Universidad Iberoamericana, México (1991). 
22) “Heráclito y el Tractatus”. En: Revista de Filosofía № 173 vol. 25. Universidad Iberoamericana, México (1992).

23) "Status y verificación de la creencia religiosa”. En: Crítica № 170 (1992), México.

24) "Sobre la investigación en filosofía”. En: Umbral XXI. № 8. Primavera 1992 (UIA).

25) “El lenguaje religioso”. Vol.: Filosofía de la religión. En: Enciclopedia Iberoamericana de Filosofía (Madrid: Trotta, 1992).

26) "El ataque de Donnellan a la teoría de las descripciones”. En: Analogía. Año VI, No 12, México (1992).

28) "La evolución de la noción de materia en el pensamiento de Russell”. En: El concepto de materia (México: Colofón, 1992).

29) "Sociedad, lenguaje y pensamiento: reflexiones en torno a la mujer”. En: La condición de la mujer mexicana. Tomo I. UNAM y Gobierno del Estado de Puebla (1992).

30) "La intolerancia semántica de Rudolf Carnap”. En: Cuadernos de Filosofía, № 137. Buenos Aires, Argentina (1993).

31) "Presentación de la traducción del libro Philosophische Bemerkungen” (ver traducciones).

32) “Nota sobre la traducción de las Philosophische Untersuchungen de Ludwig Wittgenstein”. En: Revista de Filosofía. Año 26, № 76, Universidad Iberoamericana. México (1993).

33) “Filosofía y sistematicidad”. En: Filosofía y sistema. Cuaderno 58. Instituto de Investigaciones Filosóficas (1992).

34) "Materialismo, interaccionismo y análisis gramatical”. En: El problema de la relación mente/cuerpo. Instituto de Investigaciones Filosóficas. UNAM (1993).

35) “Los colores y su lenguaje”. En: Percepción: colores (México: Instituto de Investigaciones Filosóficas, UNAM, 1993).
36) “Lenguaje y pensamiento: meditaciones post-wittgensteinianas”. En: Revista Latina de Pensamiento y Lenguaje, № 3 ,México (1993).

37) “Conductismo y filosofía”. En: Revista Mexicana de Análisis de la Conducta. Vol.16, № 12, México (1990).

38) "El sentido de la vida: problema existencial y confusión conceptual”. En: Revista Mexicana de Análisis de la Conducta. Vol. 18, México (1992).

39) “Dos nociones de objeto en el Tractatus". En: Analogía. Año VII, No 2., México (1993).

40) “Dos concepciones del lenguaje”. En: Analogía. Año VIII, No2, México (1994). Reimpreso en Algunas perspectivas de la filosofía actual en méxico (México: Universidad Iberoamericana, 1996).

41) “La filosofía de Norman Malcolm”. En: Paráfrasis. Año 1, № 1, Aguascalientes (1994).

42) “Pena capital: una re-evaluación”. En: Dilemas de la sociedad contemporánea (México: Torres Asociados, 1995).

43) "El argumento de Russell contra Frege: una exégesis”. En: Significado y Denotación (véase publicaciones, libros).

44) "Seguir una regla: resultados wittgensteinianos y especulaciones chomskyanas". En: Manuscrito. Vol. XVIII, № 2, Campinas SP/Brasil (1995).

45) "Confusiones russellianas y elucidaciones wittgensteinianas acerca de la causalidad". En: Analogía. Año X, №1, México (1996).

46) “La mente irracional”. En: Actas del Primer Congreso sobre Racionalidad. (México: UAM/Plaza y Valdés, 2000).

47) "Malinchismo filosófico y pensamiento mexicano”. En: Chicomóztoc (México: Coordinación de Humanidades, UNAM, 1997). 
48) "Progreso tecnológico y retroceso moral". En: La Tercera Revolución Industrial (México: Instituto de Investigaciones Económicas, UNAM), 1997.

49 “Pragmática y análisis gramatical”. En: Filosofía del Lenguaje II: Pragmática. Enciclopedia Iberoamericana de Filosofía (Madrid: Trotta, 1999).

50) “The presence and absence of God". En: The Rationality of Theism. Poznañ Studies in the Philosophy of the Sciences and the Humanities (Amsterdam/Atlanta: Rodopi, 2000). Reproducido en español en Concepto y Problema de Dios (México: UAM/Plaza y Valdés, 2001).

51) “El solipsismo y la realidad del pasado". En: Diálogos, N 74, Puerto Rico, Julio de 1999.

52) “Números wittgensteinianos”. En: Areté, Perú, Julio de 2000.

53) “Genética, sociedad y filosofía”. En: Ciencia, tecnología, naturaleza, cultura. En: el siglo XXI (Barcelona: Anthropos/UAM, 2000).

54) “Violencia, etica, legalidad y racionalidad”. En: Estudios sobre la violencia (México: CIESAS/Porrúa, 2002).

55) "Conocimiento y contra-ejemplos de tipo Gettier: un diagnóstico crítico”. En: Actas del Segundo Congreso sobre Racionalidad. (México: UAM/Plaza y Valdés, 2000).

56) “Bertrand Russell: filosofía y política”. En: Conciencia Mexicana, Año I, No 2. México, 1999.

57) "Espacios imaginarios y desarrollo histórico”. En: Espacios Imaginarios. Primer Coloquio Internacional. Facultad de Filosofía y Letras, UNAM, 1999.

58) “¿Qué fue la filosofía analítica”. En: Analogía. Año XIII, No 2 (México, 1999).

59) "Los sofistas, Wittgenstein y la argumentación en filosofía”. En: Tópicos, $\mathrm{N}^{\circ} 17$, 1999.
60) "Historia, derechos humanos y medicina". En: Comisión Nacional de Derechos Humanos, México 2000.

61) "La teoría de las controversias de Marcelo Dascal”. En: Manuscrito (Brasil, 2001). (en prensa).

62) "Wittgensteinian considerations about time". En: Wittgenstein, from a new point of view (Berlin: Peter Lang, 2002).

63) “Anscombe sobre la sustancia”. En: Tópi$\cos \mathrm{N}^{\circ} 21,2001$.

64) "Razón versus fe: falso dilema y conflicto dañino”. En: Fe y Razón Hoy (México: UAM/Plaza y Valdés, 2002).

65) “El Tractatus y las actitudes proposicionales”. En: Subjetividad, representación y realidad. Editado por G. Hurtado (Puebla: Benemérita Universidad Autónoma de Puebla, 2001).

66) "Tres concepciones de estructura profunda: reflexiones metodológicas”. En: Segmentos Año 1/ Núm. 1 (Guadalajara: Universidad de Guadalajara, 2001).

67) “Algunas aclaraciones. En: torno al concepto de emoción”. En: Acta Comportamentalia, Revista Latina de Análisis del Comportamiento Vol. 9, Monográfico (Guadalajara: Universidad de Guadalajara, 2001)

68) "Morality and psychology". En: Revista Mexicana de Análisis de la Conducta, vol. 27, $\mathrm{N}^{\circ} 2$, septiembre (Guadalajara: Universidad de Guadalajara, 2001).

69) “Notas sobre la felicidad". En: Memorias del Encuentro Internacional "Wittgenstein: 50 años después” (Bogotá: Pontificia Universidad Javeriana, 2002). (en prensa).

70) “David Hume y la teoría del gusto”. En: Ritmos y ucronías. Editado por Marie Noel Lapoujade. Memorias del III Congreso Internacional de Estética (Puebla: Benemérita Universidad Autónoma de Puebla, 2002). (en prensa). 
71) "Russell y Wittgenstein sobre contradicciones y paradojas”. En: Teoría, № 13 (México: Facultad de Filosofía y Letras, 2002).

72) “Frege y el Tractatus”. En: Ensayos en torno a Frege (Chihuahua, 2002). (en prensa).

73) "Karl Popper y el marxismo: somera revisión de un gran fraude intelectual” (Chihuahua, 2002).

\section{Reseñas}

1) Names and descriptions (Leonard Linsky) Crítica No 34 (1980), México (Hay traducción al polaco. Studia Filozoficzne No 112 , 1980, Polonia).

2) Metaphysics and common-sense (A. J. Ayer) Dianoia (1980), México.

3) Essays on Bertrand Russell (E. D. Klemke, ed.). Dianoia (1980), México.

4) Wittgenstein's philosophy of mathematics (V. H. Klenk). Crítica No 37 (1981), México.

5) Wittgenstein and religious belief (W. D. Hudson). Crítica No 38 (1981), México.

6) Marx and Wittgenstein. Social praxis and social explanation. (D. Rubinstein). Dianoia (1982), México.

7) Metaphysics: its structure and function (S. Korner). Revista de Filosofía № 56 (1986), México.

8) Necessity and language (A. Ambrose and M. Lazerowitz). Crítica № 53 (1986), México.

9) Investigating Wittgenstein (M. and J. Hintikka). Crítica No 55 (1987), México.

10) Wittgenstein on rules and private language (S. Kripke). Revista de Filosofía № 11 (1988), Argentina.

11) The legacy of Wittgenstein (A. Kenny). Theoria. Año III, Nos 7-8-9 (1987-1988) San Sebastián, España.
12) Wittgenstein: a critique (A. N. Findlay). Crítica No 61 (1989), México.

13) The coherence theory of truth (R. Walker) Crítica No 62 (1989), México.

14) Wittgenstein: a life. Young Wittgenstein (B. F. McGuinness). Contextos Vol.VII/13 (1989), España.

15) Science, philosophy and human behavior in the Soviet Union (L. R. Graham). Dianoia (1989), México.

16) Re-reading Russell. Essays on Bertrand Russell's metaphysics and epistemology (C. W. Savage and C. C. Anderson, eds.). Crítica No 64 (1990), México.

17) Invitation à la lecture de Wittgenstein. (G. Granger). Critica No 67 (1991), México.

18) Philosophical perspectives, 5. Philosphy of religion (editado por James E. Tomberlin). Crítica No 71 (1992), México.

19) The norm of truth. An introduction to the philosophy of logic (P. Engel). Crítica $\mathrm{N}^{\circ}$ 73 (1993), México.

20) Ludwig Wittgenstein. Entre paradojas y aporías (Silvia Rivera). Manuscrito Vol. XIX, Nº1, Abril 1996, Brasil.

21) Carnap and the Vienna Circle. Empiricism and logical syntax (Ramón Cirera). Crítica № 83 (1996), México.

22) Bertrand Russell. The spirit of solitude (Ray Monk). International Journal of Philosophical Studies. Vol. 6, Number 1, March 1998, Irlanda.

23) Teorías de la verdad. Editado por J. A. Nicolás y M. J. Frápolli. Crítica Nº 92 (1999).

24) Wittgenstein's poker. The story of tenminute argument between two great philosophers (D. Edmonds y J. Eidinow). Diánoia Vol. XLVII, N 48, mayo (2002).

25) Examen crítico del programa absolutista (Víctor Hernández Márquez). Diánoia Vol. XLVII, N 48, agosto (2002). (En prensa). 
Alejandro Tomasini Bassols, «La filosofía mística de Russell y lo indecible en el Tractatus». Diánoia. Anuario de Filosofía, Año XXXVI, Núm. 36, 1990.

\section{Traducciones}

\section{Traducciones de libros}

1) Wittgenstein on rules and private language de S. Kripke (México: Instituto de Investigaciones Filosóficas, UNAM, 1989).

2) Bemerkungen über die farben de Ludwig Wittgenstein (México y Barcelona: Paidos/ Instituto de Investigaciones Filosóficas, UNAM, 1992).

3) Philosophische bemerkungen de Ludwig Wittgenstein (México: Instituto de Investigaciones Filosóficas, UNAM, 1996).

4) Collected papers de Gareth Evans (México: Instituto de Investigaciones Filosóficas, UNAM).

5) Names and descriptions de Leonard Linsky (México: Grupo Editorial Interlínea, 1998).

\section{Traducciones de artículos}

1) “Cómo derivar 'Debe’ de 'Es'” de J. Searle. Ética y Análisis. Vol. I (México: Instituto de Investigaciones Filosóficas, UNAM, 1985).

2) “Cómo no derivar 'Debe’ de 'Es'” de J. y J. Thomson. Ética y Análisis, Vol. I (México: Instituto de Investigaciones Filosóficas, UNAM, 1985).

3) “Sobre la no derivación de 'Debe' a partir de 'Es'” de A. Flew. Etica y Análisis, Vol. I (México: Instituto de Investigaciones Filosóficas, UNAM, 1985).

4) “¿Es posible la semántica?” de H. Putnam. Col. Cuadernos de Crítica No 21 (México: Instituto de Investigaciones Filosóficas, UNAM, 1983).
5) "Algunas observaciones sobre la forma lógica de L. Wittgenstein”. Cuaderno de Filosofía № 15 (México: Universidad Iberoamericana, 1991)

6) “¿Es activa la materia?” de E. MacMullin en El concepto de materia (México: Colofón, 1992).

7) "La unidad trascendental de la apercepción" de Quassim Cassam. El problema de la relación mente-cuerpo (México: Instituto de Investigaciones Filosóficas, UNAM, 1993).

\section{Participación en congresos y conferencias}

1) “La doctrina Russelliana del pensamiento". VI Simposio Internacional de Filosofía. Agosto de 1985. Instituto de Investigaciones Filosóficas, UNAM.

2) "La comprensión de las prácticas: el caso de la magia”. XI Congreso Interamericano de Filosofía. Noviembre de 1985. Guadalajara, Jalisco (México).

3) "La percepción y la noción de ver como". III Congreso Nacional de Filosofía. Noviembre de 1985. Guadalajara, Jalisco (México).

4) "La sustancia del mundo en el Tractatus". Segundo Simposio de Filosofía. Universidad Sociedad Filosofía. Mayo de 1986. Toluca, Estado de México (México).

5) “Necesidad y convención matemáticas en la filosofía de Wittgenstein”. VII Simposio de Filosofía. Agosto de 1986. Instituto de Investigaciones Filosóficas, UNAM (México).

6) “La evolución de la noción de proposición en el pensamiento de Wittgenstein”. Abril de 1988. Academia de Ciencias de la URSS, Moscú (Unión Soviética).

7) “La gramática de Wittgenstein”. XII Congreso Interamericano de Filosofía. Julio de 1989. Buenos Aires (Argentina). 
8) "Wittgenstein y la definición ostensiva". XII Congreso Interamericano de Filosofía. Julio de 1989, Buenos Aires (Argentina).

9) “Conceptos religiosos y vida religiosa”. IX Simposio de Filosofía. Septiembre de 1989. Instituto de Investigaciones Filosóficas, UNAM (México). También publicado en: Diánoia. Anuario de Filosofía, Año XXXV, Núm. 35, 1989

10) “Confusiones russellianas y elucidaciones wittgensteinianas en torno a la causalidad”. V Congreso Nacional de Filosofía. Octubre de 1989, Jalapa, Veracruz (México).

11) “Estructura profunda”. III Jornadas Internacionales de Filosofía de la Ciencia. Noviembre de 1989, Santiago de Chile (Chile).

12) “Wittgenstein y su filosofía”. Ciclo de Conferencias Judaísmo y Cultura. Noviembre de 1989. Facultad de Filosofía y Letras, UNAM (México).

13) “La evolución de la noción de materia en el pensamiento de Russell”. Simposio: Materia. Agosto de 1990. Instituto de Investigaciones Filosóficas, UNAM.

14) “Conciencia e intencionalidad”. X Coloquio de Investigación. ENEP Iztacala. (Conferencia Magistral. Medalla de Plata), México.

15) “Sociedad, lenguaje y pensamiento: reflexiones en torno a la mujer”. Congreso Nacional de la Sociedad Mexicana de Mujeres Universitarias. Noviembre de 1990. Puebla, Puebla (México).

16) “En torno a la creencia religiosa”. Noviembre de 1990. Universidad Autónoma de Puebla, Puebla (México).

17) “Wittgenstein y la Creencia Religiosa". Noviembre de 1990. Universidad Autónoma de Puebla, Puebla, México.

18) “Dos concepciones del lenguaje”. Abril de 1991. Universidad Autónoma Metropolitana (Xochimilco), México.
19) "Materialismo, interaccionismo y análisis gramatical”. Simposio: Mente/Cuerpo. Agosto de 1991. Instituto de Investigaciones Filosóficas, UNAM.

20) "Dos nociones de objeto en el Tractatus". VI Congreso Nacional de Filosofía. Octubre de 1991. Chihuahua, México.

21) "La intolerancia semántica de Rudolf Carnap”. VI Congreso Nacional de Filosofía. Octubre de 1991. Chihuahua, (México).

22) "Perspectivas de la filosofía actual en nuestra realidad”. Coloquio: La Filosofía y su enseñanza en la Facultad de Filosofía y Letras. Enero de 1992, UNAM (México).

23) "Formación general y especialización en Filosofía”. Coloquio: La Filosofía y su enseñanza en la Facultad de Filosofía y Letras. Enero de 1992, UNAM (México).

24) "Mente-cuerpo: teoría de la identidad y emergentismo”. Programa de Superación Académica. Centro de Instrumentos. UNAM (México).

25) “Geometría y experiencia”. III Coloquio Internacional de Filosofía e Historia de las Matemáticas. Junio de 1992. Facultad de Ciencias, UNAM (México).

26) “Los colores y su lenguaje”. Simposio: Percepción: Colores. Agosto de 1992. Instituto de Investigaciones Filosóficas, UNAM (México).

27) "Filosofía, neuropsicología y conductismo”. Primer Congreso Internacional sobre el Conductismo y las Ciencias de la Conducta. Octubre de 1992. Universidad de Guadalajara, Guadalajara, Jalisco (México).

28) El sentido de la vida: confusión conceptual y problema existencial. Inauguración de cursos del Colegio de Filosofía. Marzo de 1993. Universidad Autónoma de Puebla, Puebla (México).

29) "Lenguaje y pensamiento: reflexiones postwittgensteinianas”. III Reunión Nacional y 
II Internacional de Pensamiento y Lenguaje. Octubre de 1993. Querétaro, Querétaro (México).

30) “Pena capital: un re-examen”. XIII Simposio Internacional de Filosofía. Agosto de 1994. Instituto de Investigaciones Filosóficas, UNAM (Mé-xico).

31) "Pensamiento humorístico y risa cómica". IV Congreso Latini Dies. Febrero de 1995. Guadalajara, Jalisco (México).

32) "The force and weakness of beha-viouristic explanation”. Cuarto Simposio Bienal sobre la Ciencia de la Conducta. Febrero de 1996. Chapala, Jalisco (México).

33) “Números wittgensteinianos”. XIV Simposio Internacional de Filosofía. Agosto de 1995. Instituto de Investigaciones Filosóficas. Pátzcuaro, Michoacán (México).

34) "Intereses versus moralidad: el caso del aborto”. Tecnología, desarrollo Económico y Sustentabilidad. Febrero de 1996. Puebla, Puebla (México).

35) “La mente irracional”. Coloquio sobre Racionalidad. Junio de 1997. UAM-Iztapalapa (México).

36) "Espacios imaginarios y desarrollo histórico". Primer Coloquio Internacional "Espacios Imaginarios”. Noviembre de 1997. Facultad de Filosofía y Letras, UNAM (México).

37) “Compromisos con Dios”. IX Congreso Nacional de Filosofía. Febrero de 1998. Guanajuato (México).
38) “Conocimiento y contra-ejemplos de tipo Gettier: un diagnóstico crítico”. Segundo Coloquio sobre Racionalidad. Noviembre de 1998, UAM (México).

39) “Consideraciones wittgensteinianas en torno al tiempo”. XIV Congreso Mexicano y III Ibero e Interamericano de Análisis de la Conducta. Febrero de 1999. Universidad de Gua-dalajara (México).

40) "El Tractatus y las actitudes proposicionales”. XIV Congreso Interamericano de Filosofía. Agosto de 1999. Universidad de Puebla, Puebla (México).

41) “Presencia y ausencia de Dios”. Seminario Concepto y Problema de Dios. Octubre de 1999. UAM-Iztapalapa (México).

42) "Morality and psychology". VI th Biannual Symposium on the Science of Behaviour: Experimental Analyses and Conceptual Problems. Febrero de 2000. Universidad de Guadalajara, Guadalajara (México).

43) “David Hume y la teoría del gusto”. XI Congreso Nacional de Filosofía. Agosto de 2001. UNAM (México).

44) “Notas sobre la felicidad”. Coloquio: Wittgenstein, 50 años después. Noviembre de 2001. Pontificia Universidad Javeriana (Colombia).

45) “Ciencia, pseudo-ciencia y religión”. XIV Coloquio Nacional Sobre la Enseñanza de la Filosofía. Noviembre de 2002. Colima, México. 